\title{
Retraction Note to: Clinical value of circulating tumor cells for the prognosis of postoperative transarterial chemoembolization therapy
}

\author{
Jian-wen Huang • Bing Liu $\cdot$ Bao-shan Hu • \\ Yong $\mathrm{Li} \cdot \mathrm{Xu} \mathrm{He} \cdot$ Wei Zhao $\cdot$ You-bing Zheng $\cdot$ \\ Li-gong Lu
}

Published online: 17 October 2014

(C) Springer Science+Business Media New York 2014

\section{Retraction Note to: Med Oncol (2014) 31:175 \\ DOI 10.1007/s12032-014-0175-5}

Article "Clinical value of circulating tumor cells for the prognosis of postoperative transarterial chemoembolization therapy" by Jian-wen Huang, Bing Liu, Bao-shan Hu, Yong Li, Xu He, Wei Zhao, You-bing Zheng, Li-gong Lu, published in Volume 31, Issue 9, DOI: 10.1007/s12032014-0175-5 has been retracted at the request of the authors. The author who was responsible for circulating tumor cells counting mistook some TACE group samples for control group samples in collecting and could not recall which samples were in the wrong group.

The authors apologize for any inconvenience this might have caused.

The online version of the original article can be found under doi:10.1007/s12032-014-0175-5.

J. Huang · B. Hu · Y. Li - X. He - W. Zhao - Y. Zheng ·

L. Lu (ه)

Department of Interventional Radiology, Cancer Center,

Guangdong General Hospital, Guangdong Academy of Medical

Sciences, Guangzhou, China

e-mail: luligong1969@163.com

B. Liu

Department of Interventional Radiology, Cancer Center,

Guangdong Cardiovascular Institute, Guangdong General

Hospital, Guangdong Academy of Medical Sciences,

Guangzhou, China 